\title{
THE SHORT-RANGE WAKEFIELDS IN THE BTW ACCELERATING STRUCTURE OF THE ELETTRA LINAC
}

\author{
P. Craievich*, G. D’Auria, Sincrotrone Trieste, Trieste, Italy \\ T. Weiland, I. Zagorodnov ${ }^{\dagger}$, Technische Universitaet Darmstadt, Darmstadt, Germany
}

\begin{abstract}
Future FEL operations in the ELETTRA LINAC require a high quality beam with an ultra short bunch. The knowledge of the short-range wakefields in the backward traveling wave (BTW) accelerating structure is needed to predict the beam quality in term of the single bunch energy spread and emittance. To calculate the effect of the longitudinal and transverse wakefields we have used the time domain numerical approach with a new implicit scheme for calculation of wake potential of short bunches in long structure $[1,2]$. First the wake potentials of the BTW structure are numerically calculated for very short bunches, than an analytical approximations for wake functions in short range are obtained by fitting procedures based on analytical estimations. Finally the single bunch energy spread induced by short-range longitudinal wakefields is analyzed for the first phase of the project FEL-I (up to $40 \mathrm{~nm}$ ).
\end{abstract}

\section{INTRODUCTION}

The FERMI@ELETTRA project aims to construct a single-pass FEL user-facility in the spectral range 100-10 $\mathrm{nm}$ using the exiting normal conducting $1.0 \mathrm{GeV}$ linac. Figure 1 shows the proposed machine layout for the two phase of the project: FEL-I (100-40 nm) and FEL-II (40$10 \mathrm{~nm})$ [3].

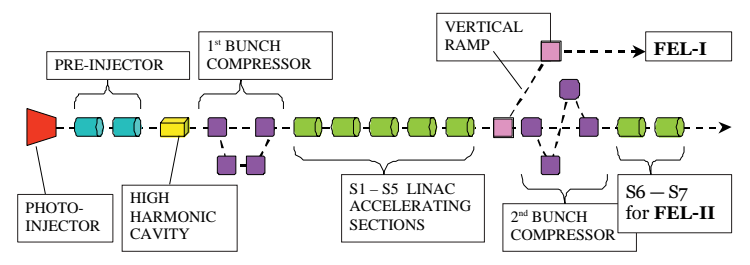

Figure 1: Schematic layout of the configuration for the FEL-I and FEL-II stages.

The new scheme foresees the installation of an RF photocathode gun [4], providing a high quality electron beam, with parameters directly related to those required at the entrance of the undulator lines [3]. At the photoinjector exit the beam is further accelerated with a $100 \mathrm{MeV}$ preinjector, composed by two $3 \mathrm{~m}$ accelerating sections with focusing solenoids, that get the beam out of the space-charge energy domain and creates an energy-position correlation for

\footnotetext{
*paolo.craievich@elettra.trieste.it

$\dagger$ This work was supported in part by the Deutsche Forschungsgemeinschaft under contract WE1239/22
}

bunch compression. After the first magnetic chicane, five accelerating structures of the exiting linac, each equipped with an RF pulse compression system (SLED), allow the beam to reach the energy required by FEL-I, $700 \mathrm{MeV}$. In total the present linac includes seven $6 \mathrm{~m}$ accelerating sections, the remaining two accelerating sections will be located after the second bunch compression and will be used for the FEL-II to rise the beam energy up to $1 \mathrm{GeV}$. Each accelerating section is a backward traveling wave (BTW) structure composed by 162 nose cone cavities magnetically coupled and operated in the $3 / 4 \pi$ mode. In this scenario, to avoid undesirable beam degradations, in term of energy spread and emittance, the wakefileds effects have to be carefully considered. We have studied the longitudinal and transverse cases using the time domain code ECHO with a new implicit scheme for the calculation of the wake potentials of short bunches in long structures [1,2]. We have considered the wakefields evolution for bunches of different lengths passing through a single cell, a multi-cell and a complete accelerating structure [5]. This paper reports the longitudinal and transverse wakefields calculations for a complete BTW accelerating structure and the single bunch energy spread induced by short-range longitudinal wakefields for the FEL-I. A preliminary optimization of the energy spread has been carried out by varying the energy gain and RF phase of the accelerating structure.

\section{LONGITUDINAL WAKE FUNCTION OF THE BTW STRUCTURE}

The wake potentials of Gaussian bunches with length ranging from $1000 \mu \mathrm{m}$ to $50 \mu \mathrm{m}$ are calculated for a whole BTW accelerating structure. In figure 2 the calculated longitudinal wake potentials (solid lines) are reported. To find an analytical approximation of the wake function we have chosen a combination of periodic [7] and one cell [6] dependence since the BTW structure can be treated as a periodic structure of finite length. From the fit of the numerical wake potentials we have obtained an analytical expression approximating the wake function (in $V / p C$ ):

$$
w 1_{\|}^{0}(s)=A_{01} e^{-\sqrt{s / s_{0}}}+\frac{A_{02}}{\sqrt{s}}
$$

where $A_{01}=7300, s_{0}=3.2 \cdot 10^{-4}$ and $A_{02}=3.4$.

Figure 2 shows the longitudinal wake function (1) (black dotted line), which tends for small $\mathrm{s}$ to be an envelope function to the wakes and fits the numerical results up to $1.5 \mathrm{~mm}$. To find an analytical approximation up to $5 \mathrm{~mm}$, we have added to expression (1) an additional term with 


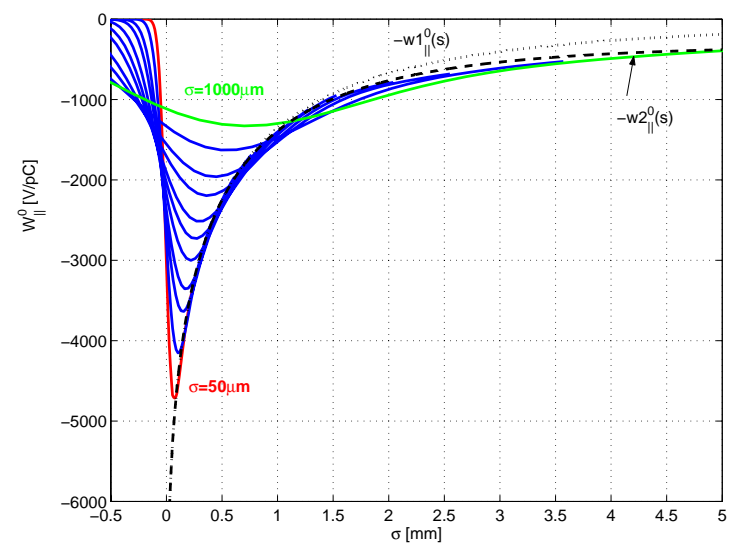

Figure 2: Longitudinal wake potentials (solid lines) and longitudinal wake functions (1)(black dotted line) and (2) (black dashed line) of the BTW structure.

$\sqrt{s}$ dependence. Taking into accoutn the last correction term the fit gives the following analytical expression for the wake function:

$$
w 2_{\|}^{0}(s)=A_{01} e^{-\sqrt{s / s_{0}}}+\frac{A_{02}}{\sqrt{s}}+A_{03} \sqrt{s}
$$

where $A_{01}=7450, s_{0}=3.1 \cdot 10^{-4}, A_{02}=3$ and $A_{03}=$ 3000 .

The previous relation approximates the longitudinal wake function on a wider range compared to expression (1). Figure 2 shows the longitudinal wake function (2) (black dashed line) that tends to be an envelope function to the wakes up to $5 \mathrm{~mm}$. Figure 3 presents the calculated longitudinal wake potentials (blue solid lines) together with analytical approximation (2) (red dashed lines). A more detailed analysis of figures 2 and 3 shows that the analytical expression 2 approximates very well the longitudinal wake function up to $5 \mathrm{~mm}$.

\section{TRANSVERSE WAKE FUNCTION OF THE BTW STRUCTURE}

Figure 4 shows the calculated transverse wake potentials (solid lines) for different bunch length $\sigma$. As in the previous case, the BTW structure is treated as a periodic structure of finite length and to find an analytical approximation for the transverse wake function, a combination of periodic [7] and one cell [6] dependence was chosen. The expression for the wake function is obtained with a fit of the numerical wake potentials (in $V / p C / m$ ):

$$
w_{\perp}^{1}(s)=A_{11}\left[1-\left(1+\sqrt{\frac{s}{s_{1}}}\right) e^{-\sqrt{s / s_{1}}}\right]+A_{12} \sqrt{s}
$$

where $A_{11}=1.7 \cdot 10^{5}, s_{1}=1.2 \cdot 10^{-4}$ and $A_{12}=8.5 \cdot 10^{4}$.

Figure 4 shows the transverse wake function (3) (black dashed line), which tends to be envelope function for the

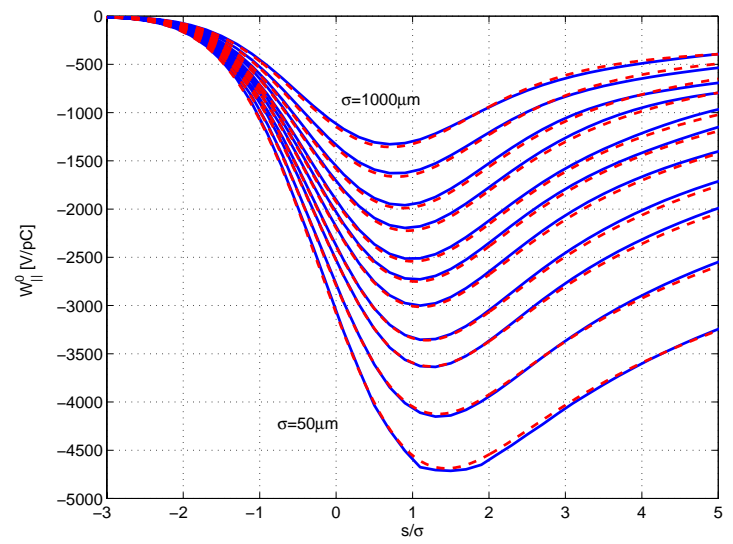

Figure 3: Longitudinal numerical (blue solid lines)and analytical (red dashed lines) wake potentials of the BTW structure.

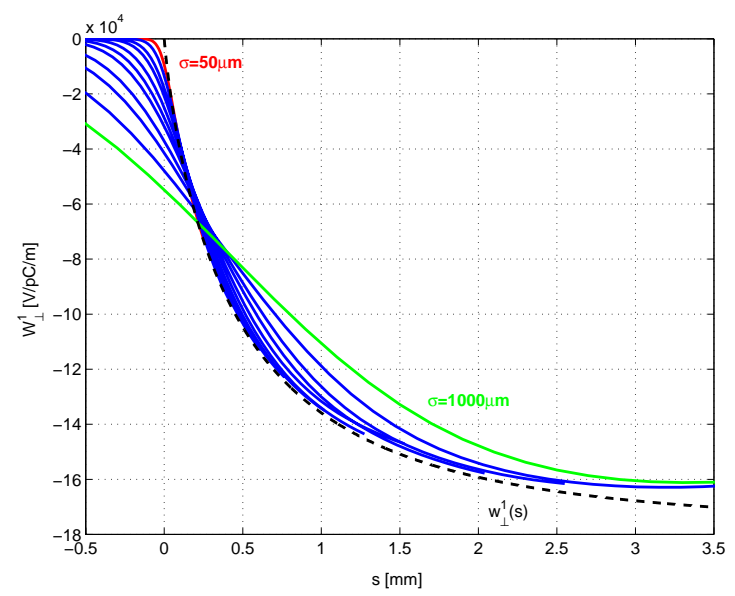

Figure 4: Transverse wake potentials (solid lines) and transverse wake functions (black dashed line) of the BTW structure.

wakes up to distance $s=2 \mathrm{~mm}$ after the bunch center. Figure 5 plots the calculated transverse wake potentials (blue solid lines) together with their analytical approximations (3) (red dashed lines). For transverse case no additional term is introduced and the wake function fits the results up to $2 \mathrm{~mm}$.

\section{SINGLE BUNCH ENERGY SPREAD IN BTW STRUCTURE}

The first phase of FERMI foresees a bunch acceleration up to $700 \mathrm{MeV}$ with bunch length $\sigma=120 \mu \mathrm{m}$ and total charge $\mathrm{Q}=1 \mathrm{nC}$. At the exit of each accelerating module, the single bunch energy spread is determined by the RF accelerating fields produced by the external generator and the wakefields excited by the beam in the accelerating structure. As already shown in [8], for Gaussian bunch the 


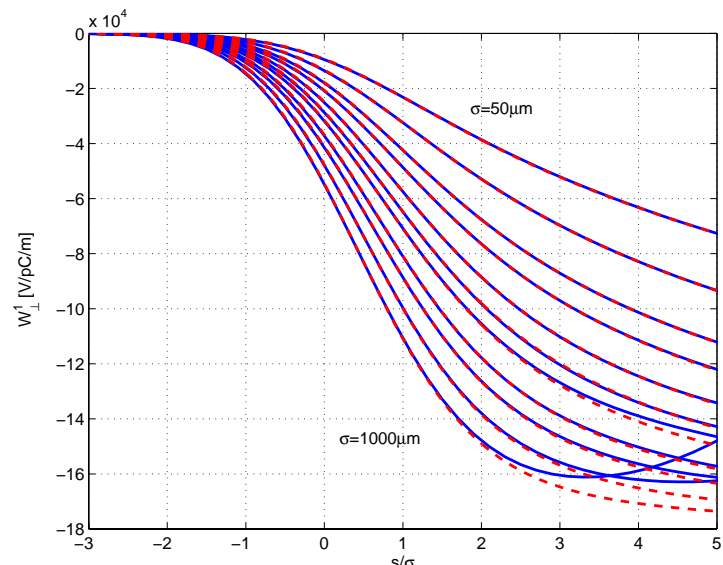

Figure 5: Transverse numerical (blue solid lines)and analytical (red dashed lines) wake potentials of the BTW structure.

RMS energy spread can be evaluated by knowing four integral parameters of the wakefields: the loss factor $K_{\|}$, the average wake energy spread $\sqrt{\Delta W^{2}}$, the cosine-Fourier part $I_{c o s}$ and the sine-Fourier part $I_{s i n}$. The last two parameters are needed to take into account the correlation between the wake potentials and the accelerating voltage [8]. Table 1 summarizes the four integral parameters for bunches of different length obtained with numerical time domain simulation (a), and analytical calculations (b) using (1): an excellent agreement between the two sets of data is shown. Figures 6 and 7 show the relative energy spread $\Delta U /\langle U\rangle$ and relative average energy gain $\langle U\rangle / U_{0}$ as a function of the RF phase $\phi$, for different energy gain per section $U_{0}$. The calculations have been made under the assumption that the energy spread is negligible with respect to the total energy gain for acceleration section and considering a maximum energy gain for section up to $170 \mathrm{MeV}$. We can see that the average energy gain at optimum phase $\phi_{\text {opt }}$ is approximately $27 \%$ lower than the corresponding value on crest, $\phi=0^{\circ}$, for each value of the peak of the acceleration voltage (figure 7). Table 2 contains the relative energy spread when the beam is accelerated on crest, RF phase $\phi=0^{\circ}$, and the minimum relative energy spread at the optimum phase $\phi_{\text {opt }}$. These parameters are given for different peak of the accelerating voltage. The relative energy spread decrease with the increase of the accelerating voltage. With an maximum energy gain of $170 \mathrm{MeV}$ the minimum relative energy spread is about 2 times lower than the corresponding at $\phi=0^{\circ}$.

To obtain the required $700 \mathrm{MeV}$ of FEL-I, the BTW strucutres have to be operated with a gradient of $21 \mathrm{MV} / \mathrm{m}$ [9]. Figure 8 shows different behaviors of the relative energy spread as a function of the electric filed gradient (changing the RF phase from $-50^{\circ}$ to $10^{\circ}$ ) for different peak accelerating voltage $U_{0}$, for a bunch with length $\sigma=120 \mu m$ with a charge $Q=1 n C$. Considering the
Table 1: Numerical and analytical integral parameters of the wake potentials of Gaussian bunches in the BTW structure.

\begin{tabular}{|c|c|c|c|c|}
\hline $\begin{array}{c}\sigma \\
{[\mu m]}\end{array}$ & $\begin{array}{c}K_{\|} \\
{[V / p C]}\end{array}$ & $\begin{array}{c}I_{\text {cos }} \\
{[V / p C]}\end{array}$ & $\begin{array}{c}I_{\text {sin }} \\
{[V / p C]}\end{array}$ & $\begin{array}{c}\sqrt{\Delta W^{2}} \\
{[V / p C]}\end{array}$ \\
\hline \multicolumn{5}{|c|}{ Numerical integral parameters (a) } \\
\hline 50 & -2861.7 & -0.001477 & -4.368020 & 1459.8 \\
120 & -2298.3 & -0.009598 & -7.860948 & 1118.4 \\
150 & -2145.5 & -0.015227 & -8.964911 & 1029.0 \\
\hline \multicolumn{5}{|c|}{ Analytical integral parameters (b) } \\
\hline 50 & -2861.2 & -0.001546 & -4.307913 & 1441.2 \\
120 & -2295.4 & -0.009476 & -7.831107 & 1112.5 \\
150 & -2145.8 & -0.014982 & -8.961020 & 1026.3 \\
\hline
\end{tabular}

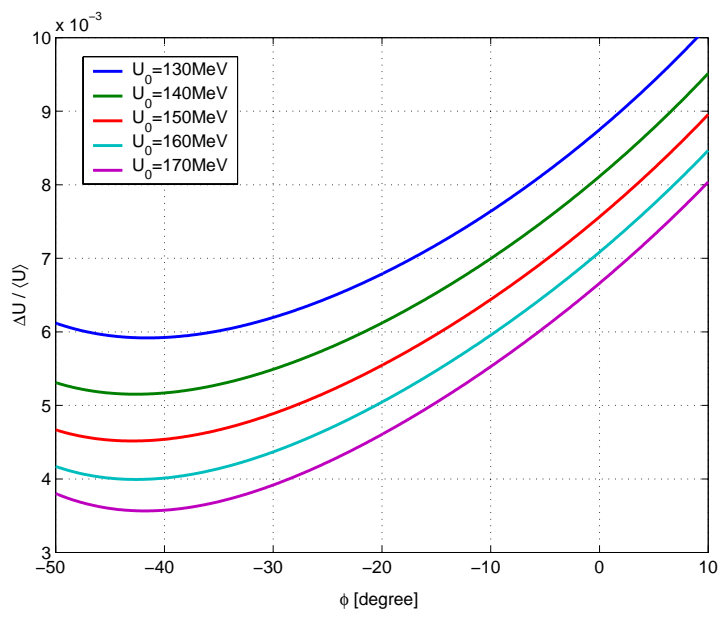

Figure 6: Relative energy spread as a function of the RF phase in the BTW structure (bunch length $\sigma=120 \mu \mathrm{m}$ and $Q=1 n C$ ).

$21 \mathrm{MV} / \mathrm{m}$ required, we can see that the relative energy spread is near its minimum value only for $U_{0}=170 \mathrm{MeV}$. With lower values of $U_{0}$ the relative energy spread don't get its minimum value through the tuning of RF phase.

\section{CONCLUSION}

Calculations of the wakefields for short bunches passing through a complete BTW accelerating structure have been presented. The short-range longitudinal and transverse wake potentials are been calculated in time domain with the code ECHO. From the numerical results analytical approximations of the point-charge wake functions were found. For the analytical model we have chosen as a combination of periodic and one-cell dependences. In the longitudinal case the term that describes the finite structure (one cell behavior) is very small compared to the periodic structure term. Hence in range of $\sigma$ we have considered, the longitudinal wakes shows mainly a periodic structure behavior. In addition, for better fitting of the data up to 5 
Table 2: Energy gain and energy spread in the BTW structure (bunch length $\sigma=120 \mu \mathrm{m}$ and $Q=1 \mathrm{nC}$ ).

\begin{tabular}{|c|c|c|c|}
\hline $\begin{array}{c}U_{0} \\
{[\mathrm{MeV}]}\end{array}$ & RF phase & $\begin{array}{c}\langle U\rangle \\
{[M e V]}\end{array}$ & $\begin{array}{c}\Delta U /\langle U\rangle \\
\%\end{array}$ \\
\hline 130.0 & $\phi=0.0^{\circ}$ & 127.70 & 0.87 \\
& $\phi_{o p t}=-41.6^{\circ}$ & 94.96 & 0.59 \\
\hline 140.0 & $\phi=0.0^{\circ}$ & 137.70 & 0.81 \\
& $\phi_{o p t}=-42.6^{\circ}$ & 100.69 & 0.51 \\
\hline 150.0 & $\phi=0.0^{\circ}$ & 147.70 & 0.76 \\
& $\phi_{o p t}=-43.0^{\circ}$ & 107.48 & 0.45 \\
\hline 160.0 & $\phi=0.0^{\circ}$ & 157.70 & 0.71 \\
& $\phi_{o p t}=-42.6^{\circ}$ & 115.44 & 0.40 \\
\hline 170.0 & $\phi=0.0^{\circ}$ & 167.70 & 0.67 \\
& $\phi_{o p t}=-41.8^{\circ}$ & 124.47 & 0.36 \\
\hline \multicolumn{3}{|c}{}
\end{tabular}

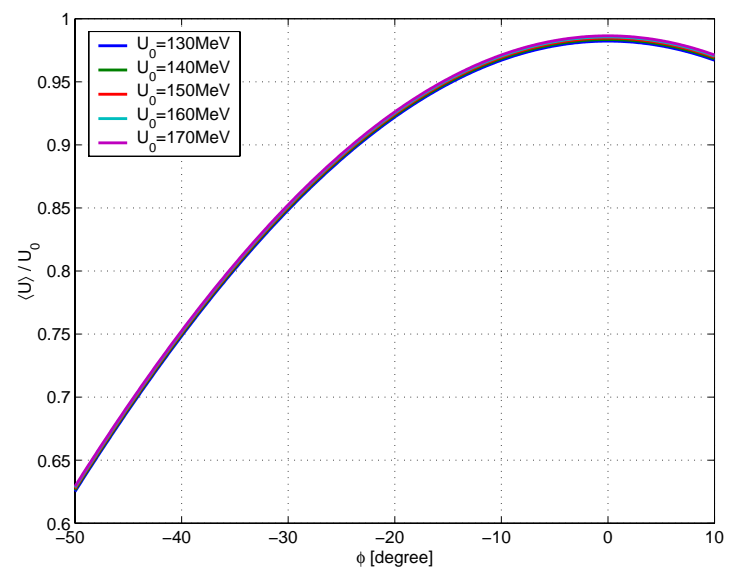

Figure 7: Relative energy gain as a function of RF phase in the BTW structure (bunch length $\sigma=120 \mu \mathrm{m}$ and $Q=$ $1 n C)$.

$\mathrm{mm}$, we have used an additional term in the model. Furthermore, the single bunch energy spread induced by longitudinal wakefields has been analyzed for the first phase of the project FEL-I. We have seen that the wakefield effects can be compensated by shifting the bunch injection phase to optimum values, decreasing the energy gain of about $27 \%$. For the $700 \mathrm{MeV}$ energy design of FEL-I and a peak gradient of $27.8 \mathrm{MV} / \mathrm{m}$ (170 Mev energy gain for section) this means to operate at $\phi=-41.8^{\circ}$ and a gradient of $21 \mathrm{Mv} / \mathrm{m}$.

\section{REFERENCES}

[1] A. Novokhatski, M. Timm, T. Weiland, "Transition Dynamics of the Wake Fields of Ultra Short Bunches", Proceeding of the ICAP 1998, California, USA.

[2] I. Zagorodnov, T. Weiland, "Calculation of Transverse Wake Potential for Short Bunches”, ICAP 2002.

[3] "FERMI@ELETTRA machine project update", Sincrotrone Trieste, 2003.

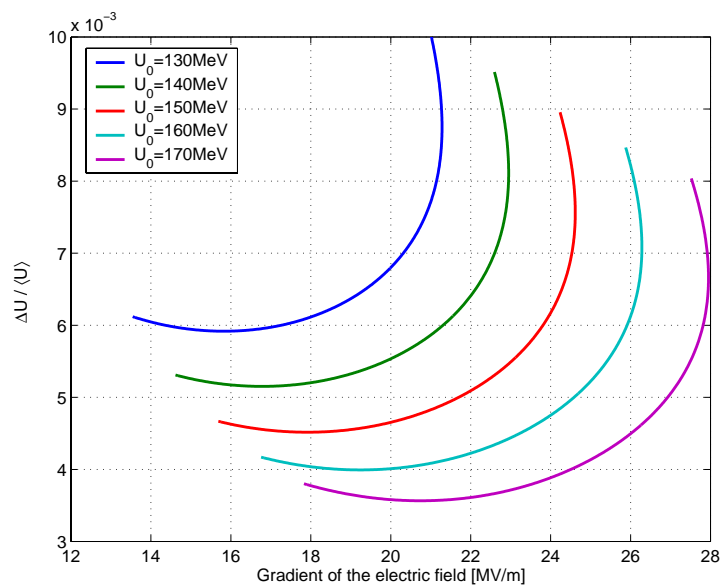

Figure 8: Relative energy spread as a function of the gradient of the electric field in the BTW structure (bunch length $\sigma=120 \mu m$ and $Q=1 n C)$.

[4] V.A. Verzilov et al., "Photo-injector study for the ELETTRA linac FEL”, FEL-2003, Tsukuba, Japan.

[5] P. Craievich, T. Weiland, I. Zagorodnov, Report ST/M-04/02, Sincrotrone Trieste, 2004.

[6] K. Bane, M. Sands, "Wakefields of very short bunches in an accelerating cavity", SLAC-PUB-4441, 1987.

[7] K. Bane, "Shortrange dipole wakefields in accelerating structures for the NLC", SLAC-PUB-9663, LCC-0116, 2003.

[8] A. Novokhatski, M. Timm and T. Weiland, "Single Bunch Energy Spread in the TESLA Cryomodule", TESLA Report 99-16, 1999.

[9] S. Di Mitri et al., "Start-to-end simulation for the FERMI project at ELETTRA", this conference. 